\title{
Pulmonary imaging in coronavirus disease 2019 (COVID-19): a series of 140 Latin American children
}

\author{
Carlos F. Ugas-Charcape ${ }^{1}$ (D) María Elena Ucar ${ }^{2}$ - Judith Almanza-Aranda ${ }^{3}$ • Emiliana Rizo-Patrón ${ }^{4}$. \\ Claudia Lazarte-Rantes ${ }^{1}$. Pablo Caro-Domínguez ${ }^{5} \cdot$ Lina Cadavid $^{6}$. Lizbet Pérez-Marrero ${ }^{7}$. Tatiana Fazecas ${ }^{8}$. \\ Lucía Gomez $^{9}$. Mariana Sánchez Curiel ${ }^{10}$ - Walter Pacheco ${ }^{11}$ • Ana Rizzi ${ }^{12}$ • Andrés García-Bayce ${ }^{13}$. \\ Efigenia Bendeck $^{14}$. Mario Montaño ${ }^{15} \cdot$ Pedro Daltro $^{16}$. José D. Arce-V ${ }^{17}$
}

Received: 2 October 2020 / Revised: 4 February 2021 / Accepted: 16 March 2021 / Published online: 1 April 2021

(C) The Author(s), under exclusive licence to Springer-Verlag GmbH Germany, part of Springer Nature 2021

\begin{abstract}
Background The severe acute respiratory syndrome coronavirus 2 (SARS-CoV-2), which resulted in the worldwide coronavirus disease 2019 (COVID-19) pandemic of 2020, has particularly affected Latin America.

Objective The purpose of the study was to analyze the imaging findings of pulmonary COVID-19 in a large pediatric series. Materials and methods Children with SARS-CoV-2 infection confirmed by either quantitative reverse transcriptionpolymerase chain reaction from nasopharyngeal swabs or presence of circulating immunoglobulin $\mathrm{M}$ (IgM) antibodies and who underwent chest radiograph or CT or both were included in this retrospective multicenter study. Three pediatric radiologists independently reviewed radiographs and CTs to identify the presence, localization, distribution and extension of pulmonary lesions.

Results We included 140 children (71 female; median age 6.3 years, interquartile range 1.6-12.1 years) in the study. Peribronchial thickening (93\%), ground-glass opacities (79\%) and vascular engorgement (63\%) were the most frequent findings on 131 radiographs. Ground-glass opacities $(91 \%)$, vascular engorgement $(84 \%)$ and peribronchial thickening $(72 \%)$ were the most frequent findings on 32 CTs. Peribronchial thickening (100\%), ground-glass opacities $(83 \%)$ and pulmonary vascular engorgement $(79 \%)$ were common radiograph findings in asymptomatic children $(n=25)$. Ground-glass opacity and consolidation were significantly higher in children who needed intensive care admission or died (92\% and 48\%), in contrast with children with a favorable outcome ( $71 \%$ and $24 \%$, respectively; $P<0.05$ ).
\end{abstract}

Carlos F. Ugas-Charcape

cugas@insnsb.gob.pe

1 Department of Diagnostic Imaging, Instituto Nacional de Salud del Niño San Borja, Av. Javier Prado Este 3101, 15037 Lima, Peru

2 Servicio de Diagnóstico por Imágenes, Hospital de Niños Sor María Ludovica, La Plata, Argentina

3 Servicio de Imagenología, Salud Digna, Ciudad de México, Mexico

4 Unidad de Desarrollo de Investigación, Tecnologías y Docencia, Instituto Nacional de Salud del Niño San Borja, Lima, Peru

5 Unidad de Radiología Pediátrica, Servicio de Radiología, Hospital Universitario Virgen del Rocío, Sevilla, Spain

6 Radiology Department, Hospital Pablo Tobón Uribe - IMEDI, Medellín, Colombia

7 Departamento de Imágenes, Facultad de Medicina Clínica Alemana, Universidad del Desarrollo, Santiago de Chile, Chile

8 Department of Diagnostic Imaging, Hospital Municipal Jesus, Alta Excelência Diagnóstica/DASA,

Clínica de Diagnóstico por Imagem/DASA, Rio de Janeiro, Brazil
9 Servicio de Imagen, Hospital Pediátrico Baca Ortiz, Quito, Ecuador

10 Department of Diagnostic Imaging, Hospital Infantil de México Federico Gómez, Mexico City, Mexico

11 Servicio de Radiología e Imágenes Médicas, Hospital María Especialidades Pediátricas, Tegucigalpa, Honduras

12 Departamento de Diagnóstico por Imágenes, Hospital de Pediatria Prof. Dr. Juan P. Garrahan, Buenos Aires, Argentina

13 Department of Imaging, Centro Hospitalario Pereira Rossell, Montevideo, Uruguay

14 Departamento de Radiología e Imágenes, Hospital Nacional de Niños “Benjamin Bloom,”, San Salvador, El Salvador

15 Servicio de Diagnóstico por Imágenes, Hospital Santa Cruz C.P.S., Santa Cruz de la Sierra, Bolivia

16 Alta Excelência Diagnóstica/DASA and Clínica de Diagnóstico por Imagem/DASA, Rio de Janeiro, Brazil

17 Servicio de Radiología e Imágenes, Clínica Santa María, Santiago, Chile 
Conclusion Asymptomatic children and those with mild symptoms of COVID-19 showed mainly peribronchial thickening, ground-glass opacities and pulmonary vascular engorgement on radiographs. Ground-glass opacity and consolidation were more common in children who required intensive care admission or died.

Keywords Chest $\cdot$ Children · Computed tomography $\cdot$ Coronavirus disease $2019 \cdot$ Lungs $\cdot$ Pulmonary $\cdot$ Radiography $\cdot$ Severe acute respiratory syndrome coronavirus 2

\section{Introduction}

Severe acute respiratory syndrome coronavirus 2 (SARSCoV-2), which causes coronavirus disease 2019 (COVID19), represents an ongoing and enduring worldwide health threat [1]. Since the first reported case of COVID-19 reported in Brazil in late February 2020, the infection rate in Latin America has increased exponentially, with more than 16 million cases and 530,000 official deaths at the time of this report [2].

Although COVID-19 predominantly affects the adult population, children and young adults are not exempt from infection, and they can further increase community transmission rates [3]. Despite their higher probability of experiencing only a mild clinical course, some severe cases have been reported, including children with multifocal pneumonia that progresses to respiratory failure and multisystem immune-mediated inflammatory disease known as multisystem inflammatory syndrome in children (MIS-C) [4, 5].

The first imaging studies of people with COVID-19 included a small number of cases and were not exclusively pediatric $[6,7]$. The initial large pediatric series of cases showed that chest radiographs and CT findings in COVID-19 are nonspecific and are not helpful in differentiating this infection from other childhood lower respiratory tract infections or pneumonia $[8,9]$.

The current study is the first international multicenter investigation promoted by the Sociedad Latinoamericana de Radiología Pediátrica (SLARP), and its aim is to report on radiologic pulmonary findings in a large pediatric series.

\section{Materials and methods}

The ethics committees and institutional review boards of the Instituto Nacional de Salud del Niño San Borja (Lima, Perú), the Hospital Infantil de México Federico Gómez (México City, México) and the Hospital de Niños Sor María Ludovica (La Plata, Argentina) approved the study. The institutions in Perú and México waived the requirement for informed patient consent, and written informed consent was obtained at the hospital in Argentina. Members of the SLARP from all Latin American countries were invited to participate.

\section{Patients}

The study included consecutive pediatric patients (ages 18 years and younger) with COVID-19 infection as confirmed by real-time reverse-transcriptase polymerase chain reaction (RT-PCR) or immunoglobulin M (IgM) serology who underwent at least one thoracic imaging modality (radiography or CT) between May 15 and June 25 in 2020. We retrospectively reviewed clinical data, including demographics, symptoms, days from symptom onset to exam date, comorbidities, intensive care unit (ICU) admission, and fatal outcomes. Collaborators used a standardized data collection spreadsheet to record the clinical data from each site (Online Supplementary Material 1).

\section{Image acquisition}

Radiographs and CT images were acquired in accordance with institutional protocols of each participating center. The choice of imaging technique depended on equipment availability and clinical context, as well as regional/national needs and recommendations. Images were anonymized, coded and stored in an online digital repository (Google Drive; Google, Mountain View, CA) for assessment.

\section{Imaging interpretation}

Three pediatric radiologists (M.E.U., P.D. and J.A.), with 24, 35 and 43 years of experience in pediatric radiology, respectively, independently reviewed the images using a free Digital Imaging and Communications in Medicine (DICOM) software viewer (RadiAnt; Medixant Maciej Frankiewicz, Poznan, Poland). Despite awareness that all subjects had tested positive for infection with the SARS-CoV-2 virus, the reviewers were blinded to clinical and outcome information. Radiographs and CT images were analyzed to identify the presence of pathological findings such as peribronchial thickening, ground-glass opacities, crazy-paving pattern, consolidations, halo signs, reverse halo signs, atelectasis, linear opacities, pleural effusion and lymphadenopathies (Online Supplementary Material 2). The definitions for the findings were described by using international standard nomenclature from the Fleischner Society glossary of terms for thoracic 
imaging and adding the term "peribronchial thickening" as reported in a similar recently published series [10].

Vascular engorgement was defined as positive when pulmonary vessels were found up to the subpleural level (especially in the longitudinal vessel plane) and when their diameter was greater than the accompanying bronchus (in the axial vessel plane) (Figs. 1 and 2). Because of the high frequency of vascular engorgement, the three evaluating pediatric radiologists carried out a separate analysis to determine the presence of vascular engorgement as the predominant pattern in radiographs. We excluded children with comorbidities such as congenital heart disease and other pulmonary overflowcausing pathologies that could confound this sign.

\section{Statistical analysis}

To define the presence of a pathological finding, its localization and distribution, we required the agreement of at least two reviewers. We determined inter-rater agreement among all three evaluators for four radiographic signs (peribronchial thickening, ground-glass opacity, vascular engorgement and consolidation) and eight $\mathrm{CT}$ signs (peribronchial thickening, ground-glass opacity, consolidation, crazy-paving pattern, halo signs, reverse halo signs, vascular engorgement and linear opacities) using the Fleiss kappa statistic or Brennan-Prediger kappa coefficient in the presence of high agreement, low kappa paradox $[11,12]$.

Continuous variables were expressed as medians and interquartile ranges or as means and standard deviation; categoric variables were presented as values and percentages. We

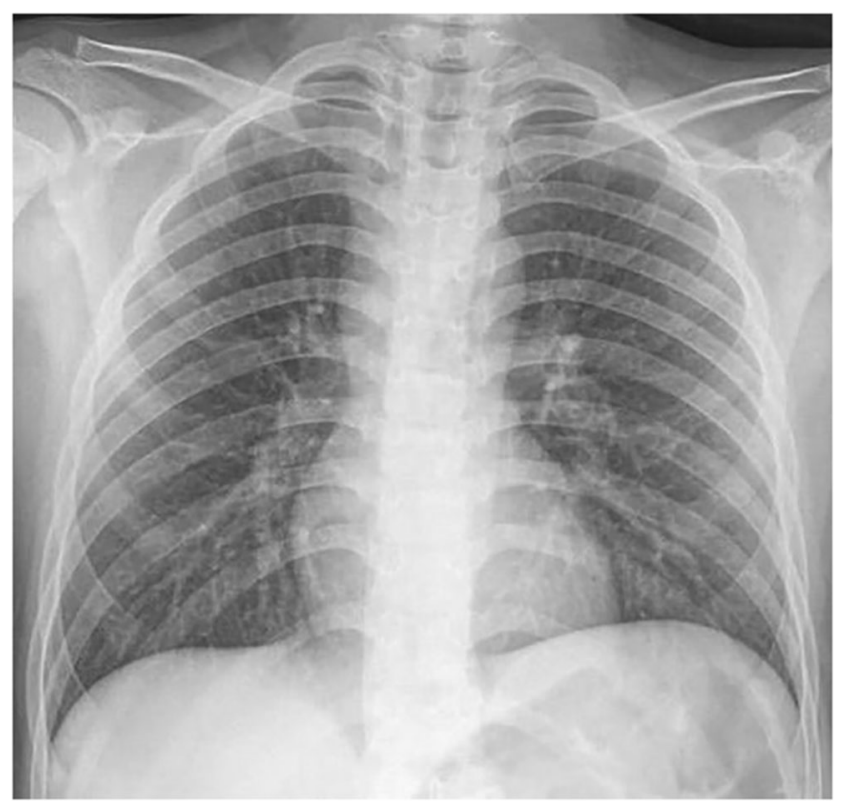

Fig. 1 Vascular engorgement on radiography in an 11-year-old boy with fever for $24 \mathrm{~h}$, previously healthy. Anteroposterior chest radiograph shows bilateral vascular engorgement in the left lower lobe pulmonary vessels reaching the subpleural surface (longitudinal vessel plane)

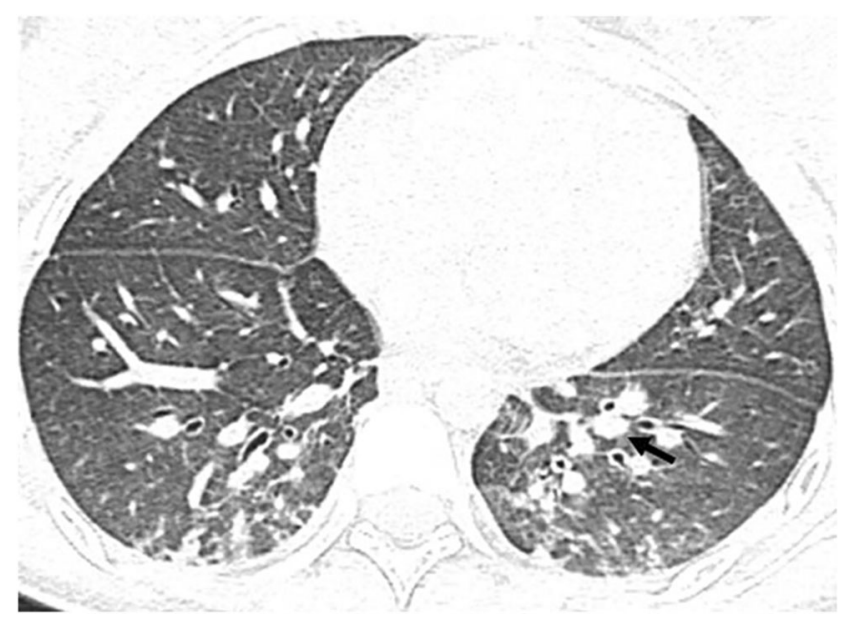

Fig. 2 Vascular engorgement on CT in an 11-year-old girl with cough and dyspnea for 7 days. Axial lung window CT image shows vascular diameter greater than the accompanying bronchus in the lower lobes (arrow)

calculated the frequencies of pathological findings for each clinical characteristic and compared them using the chisquare or Fisher exact test. The number of days from onset of symptoms until the image date for each finding was compared using the Mann-Whitney $U$ test. Two-tailed $P<0.05$ was considered statistically significant. All statistical analyses were performed with Stata 15 software (StataCorp, College Station, TX).

\section{Results}

\section{Patients and clinical findings}

The final study population included 140 children, after excluding 8 subjects with poor-quality images that were nondiagnostic. Participants included 69 boys and 71 girls, with a median age of 6.3 years, and an interquartile range of 1.612.1 years. All participants had COVID-19 infection confirmed by RT-PCR $(n=126,90 \%)$ or immunoglobulin (IgM) test $(n=14,10 \%)$. One hundred thirty-one children had radiography (108 had only radiographs), and 32 children had CT (9 had only CT). Table 1 summarizes the demographic, clinical and radiologic findings. For analytical purposes, the participants were divided into three groups: the preschool age group included children $0-6$ years old $(47.1 \%)$, the primary school age group included children $6-13$ years old (30.0\%), and the teenage group included adolescents 13-18 years old $(22.9 \%)$.

Cases were submitted from Peru $(n=47,33.6 \%)$, Mexico $(n=26,18.6 \%)$, Brazil $(n=16,11.4 \%)$, Argentina $(n=15$, $10.7 \%)$, Colombia $(n=12,8.6 \%)$, Ecuador $(n=11,7.9 \%)$, Honduras $(n=8,5.7 \%)$, Chile $(n=3,2.1 \%)$ and El Salvador $(n=2,1.4 \%)$. 
Table 1 Clinical and pulmonary findings of 140 Latin American children with coronavirus disease 2019 (COVID-19)

\begin{tabular}{|c|c|c|}
\hline Clinical features & $n=140$ & \\
\hline \multicolumn{3}{|l|}{ Age (years) } \\
\hline Mean, SD & 7.3 & 5.8 \\
\hline Median, IQR & 6.3 & $1.6-12.1$ \\
\hline \multicolumn{3}{|l|}{ Age group $n, \%$} \\
\hline $0-6$ years & 66 & 47.1 \\
\hline $6-13$ years & 42 & 30.0 \\
\hline$>13$ years & 32 & 22.9 \\
\hline Female $n, \%$ & 71 & 50.7 \\
\hline \multicolumn{3}{|l|}{ Symptoms $n, \%$} \\
\hline Fever & 81 & 57.9 \\
\hline Dyspnea & 39 & 27.9 \\
\hline Cough & 27 & 19.3 \\
\hline Tachypnea & 22 & 16 \\
\hline Tachycardia & 9 & 6.5 \\
\hline Rhinorrhea & 6 & 4.3 \\
\hline Productive cough & 5 & 3.6 \\
\hline Odynophagia & 5 & 3.6 \\
\hline No symptoms & 25 & 17.9 \\
\hline Comorbidities $n, \%$ & 92 & 65.7 \\
\hline \multicolumn{3}{|l|}{ Clinical outcome $n, \%$} \\
\hline Favorable & 87 & 62.1 \\
\hline ICU admission & 34 & 24.3 \\
\hline Mechanical ventilation & 7 & 5.0 \\
\hline Death & 12 & 8.6 \\
\hline \multicolumn{3}{|c|}{ Time from symptom onset to exam date } \\
\hline \multicolumn{3}{|c|}{ Chest radiograph (days) } \\
\hline Mean, SD & 2.1 & 2.2 \\
\hline Median, IQR & 1 & $1-3$ \\
\hline \multicolumn{3}{|l|}{ Chest CT (days) } \\
\hline Mean, SD & 8.8 & 10.1 \\
\hline Median, IQR & 6.5 & $9.5-11.5$ \\
\hline \multicolumn{3}{|l|}{ Imaging findings } \\
\hline Chest radiograph $n, \%$ & 131 & 93.6 \\
\hline Peribronchial thickening & 122 & 93.1 \\
\hline Ground-glass opacity & 103 & 78.6 \\
\hline Vascular engorgement & 82 & 62.6 \\
\hline Consolidation & 43 & 32.8 \\
\hline Pleural effusion & 8 & 6.1 \\
\hline Atelectasis & 3 & 2.3 \\
\hline Chest CT $n, \%$ & 32 & 22.9 \\
\hline Ground-glass opacity & 29 & 90.6 \\
\hline Vascular engorgement & 27 & 84.4 \\
\hline Peribronchial thickening & 23 & 71.9 \\
\hline Consolidation & 22 & 68.8 \\
\hline Halo & 12 & 37.5 \\
\hline Reverse halo & 11 & 34.4 \\
\hline Crazy paving & 10 & 31.3 \\
\hline Linear opacity & 5 & 15.6 \\
\hline Pleural effusion & 4 & 12.9 \\
\hline Nodule & 3 & 9.7 \\
\hline
\end{tabular}

$I C U$ intensive care unit, $I Q R$ interquartile range, $S D$ standard deviation

The majority of children were experiencing symptoms at the time of imaging ( $n=115,82.1 \%)$, with fever the most common $(n=81 ; 57.9 \%)$, followed by dyspnea $(n=39,27.9 \%)$ and cough $(n=27,19.3 \%)$. Indications for imaging included the assessment of SARS-CoV-2 infection in 58 children $(41.4 \%)$, diagnosis of lower respiratory tract infection in 37 children $(26.4 \%)$, evaluation of previous established comorbidities or acute disease in 31 children $(22.1 \%)$ and detection of possible complications in 10 children $(7.1 \%)$. In four children the imaging indication was not specified.

Approximately two-thirds of the children $(n=92 ; 65.7 \%)$ had underlying conditions at the time of diagnosis of SARSCoV-2 infection. Sixteen children had congenital heart disease (11.4\%), 13 had cancer $(9.3 \%)$ and 10 had an immunodeficiency disorder $(7.1 \%)$. Other underlying conditions of various etiologies were documented in the remaining 53 children (Table 2).

Of all the children in this series, $62.1 \%(n=87)$ had a good clinical course and were discharged after improvement, and $29.3 \%(n=41)$ were admitted to the ICU or underwent mechanical ventilation. Twelve participants $(8.6 \%)$ died despite resuscitation measures. We were unable to obtain clinical follow-up data for four children.

\section{Chest radiography}

All of the 131 radiographs analyzed had positive findings. Radiographs were acquired on average $2.1 \pm 2.2$ days after the onset of symptoms. The most frequent finding was peribronchial thickening in $93.1 \%(n=122)$ (Fig. 3), followed by ground-glass opacity in $78.6 \%(n=103)$ and vascular engorgement in $62.6 \%(n=82)$.

Ground-glass opacities were described as predominantly bilateral $(n=65 ; 63.1 \%)$, peripheral $(n=45 ; 43.7 \%)$ and in the lower lobes ( $n=48 ; 51.1 \%$ ) (Figs. 4 and 5$)$. Vascular engorgement was bilateral in most cases $(n=59 ; 72.0 \%)$ and was generalized in both upper and lower lobes in $44(53.6 \%)$ cases (Figs. 1, 5, 6, 7 and 8). Consolidation was found in $32.8 \%$ of radiographs $(n=43)$, usually peripheral $(n=11 ; 36.0 \%)$, unilateral $(n=21 ; 48.8 \%)$ and affecting the lower lobes $(n=27$; $62.8 \%$ ) (Fig. 9). Other findings are noted in Table 1.

Table 2 Comorbidities or associated conditions of 140 Latin American children with coronavirus disease 2019 (COVID-19)

\begin{tabular}{lll}
\hline Comorbidities or conditions & $n$ & Percentage \\
\hline None & 48 & 34.3 \\
Congenital/acquired heart disease & 16 & 11.4 \\
Cancer & 13 & 9.3 \\
Immunodeficiency disorder & 10 & 7.1 \\
Chronic pulmonary disease & 9 & 6.4 \\
Metabolic disorder/obesity & 9 & 6.4 \\
Neurologic condition & 8 & 5.7 \\
Burned patient & 6 & 4.3 \\
Orthopedic condition & 6 & 4.3 \\
Gastric/digestive system disease & 6 & 4.3 \\
Other infectious disease & 3 & 2.1 \\
Post-surgical state & 3 & 2.1 \\
Chronic renal disease & 2 & 1.4 \\
Puerperium & 1 & 0.7 \\
\hline
\end{tabular}


The frequency of ground-glass opacity was significantly higher in the preschool age group $(n=56 ; 88.9 \%)$ and lower in the teenage group ( $n=20 ; 66.7 \%, P=0.017)$. Vascular engorgement was observed significantly more frequently in primary school age children than in the preschool age and teenage groups ( $79 \%$ vs. $54 \%$ and $60 \%, P=0.036)$. Consolidation was significantly more frequent in teenagers and less frequent in preschoolers $(53.3 \%$ vs. $23.8 \%, P<0.019)$ (Table 3$)$.

Compared with asymptomatic children, consolidation was found more frequently in symptomatic children $(37.4 \%$ vs. $12.5 \%, P=0.019)$. Findings like peribronchial thickening $(100 \%)$, ground-glass opacity $(83.3 \%)$ and vascular engorgement $(79.2 \%)$ were more frequent in asymptomatic children; however, no statistical significance was found (Table 3).

In participants with a good clinical course who were not admitted to the ICU, peribronchial thickening was the most frequent imaging finding (97.6\%), followed by ground-glass opacity $(71.1 \%)$, vascular engorgement (69.9\%) and consolidation $(24.1 \%)$ (Table 3$)$. Radiographs of children with a more severe clinical course (ICU admission, mechanical ventilation or death), showed ground-glass opacity in $91.7 \%$ and peribronchial thickening in $85.4 \%$, followed by vascular engorgement in $50.0 \%$ and consolidation in $47.9 \%$ (Table 3). Therefore, radiographic findings correlated to clinical course: peribronchial thickening and vascular engorgement were the more frequent findings in children with favorable outcomes than in those who required ICU admission or died $(P=0.008$ and $P=0.023$, respectively). Consolidation and ground-glass opacity were more frequently found in children who required ICU admission or died than in those who had a milder clinical course ( $P=0.005$ and $P=0.006$, respectively). See Online Supplementary Material 3.

Consolidation was found in $28.3 \%$ of radiographs taken fewer than 7 days from clinical onset compared to $61.1 \%$ in

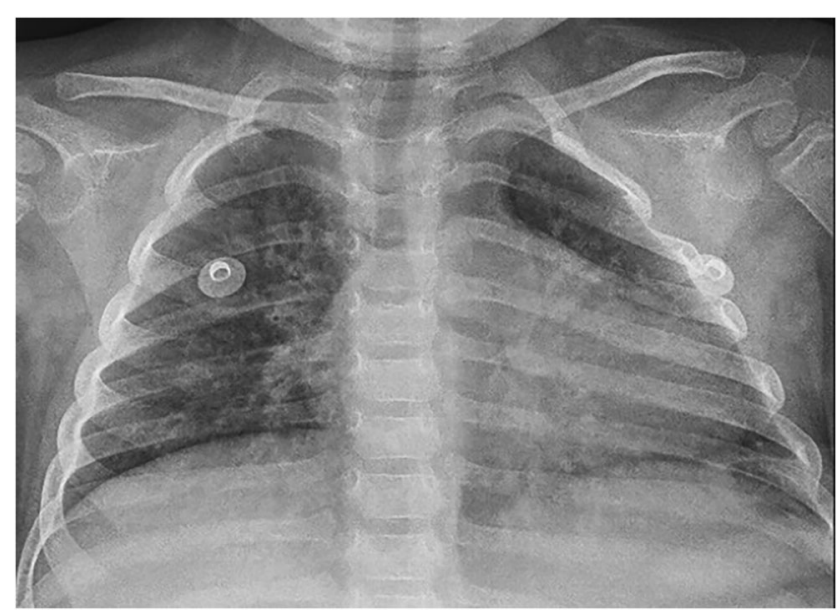

Fig. 3 Peribronchial thickening in a 2-year-old asymptomatic girl with juvenile idiopathic arthritis and immunosuppression. Anteroposterior chest radiograph shows central peribronchial thickening with peribronchial cuffing

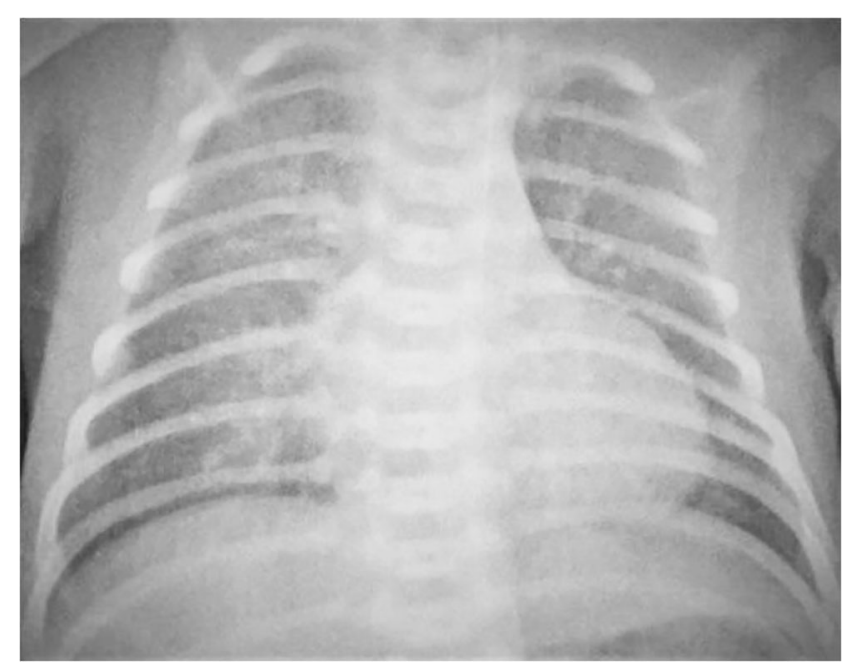

Fig. 4 Ground-glass opacities in an 8-day-old asymptomatic girl. A portable anteroposterior chest radiograph shows ground-glass opacities, peribronchial thickening and left basal atelectasis (retrocardiac)

radiographs taken more than 7 days from clinical onset $(P=0.006)$. No statistically significant association was found between the time from the onset of symptoms and other radiologic signs (Table 3 and Online Supplementary Material 3).

After excluding underlying cardiovascular disease and other conditions related to pulmonary vascular overflow $(n=17)$, vascular engorgement was the predominant pattern in 25 children (21.9\%) (Figs. 1 and 7). The pattern was most frequent in boys $(60 \%)$, in the primary school group (44\%) and in symptomatic children $(83.3 \%)$. It was present in $16.7 \%$ of asymptomatic children. Children who were imaged $<7$ days after symptom onset showed vascular engorgement

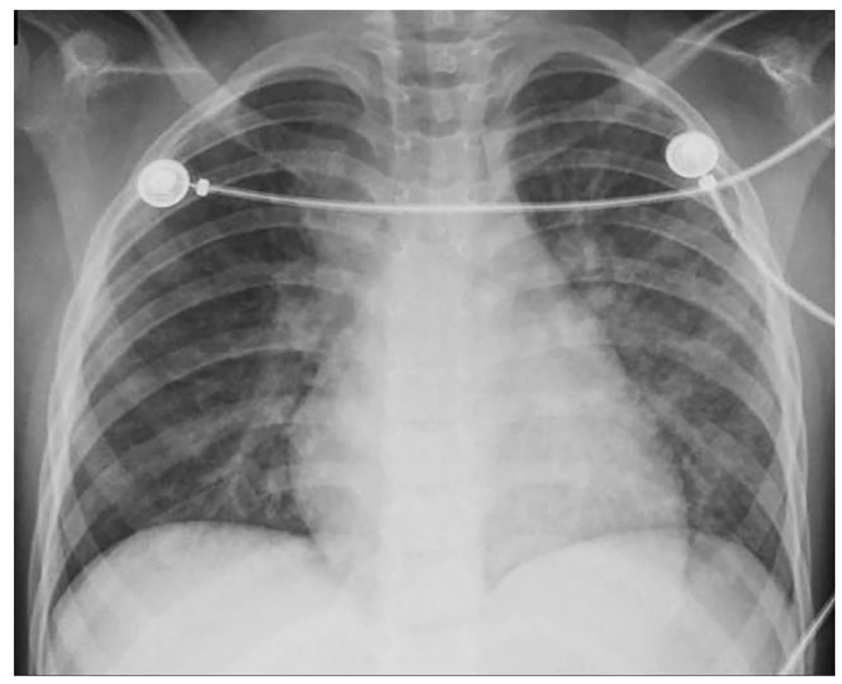

Fig. 5 Ground-glass opacities in a 6-year-old girl with leukemia. She presented with fever and respiratory distress for 2 days and was hospitalized in the intensive care unit. Portable anteroposterior chest radiograph shows central ill-defined ground-glass opacities, vascular engorgement and consolidation in the right upper lobe 


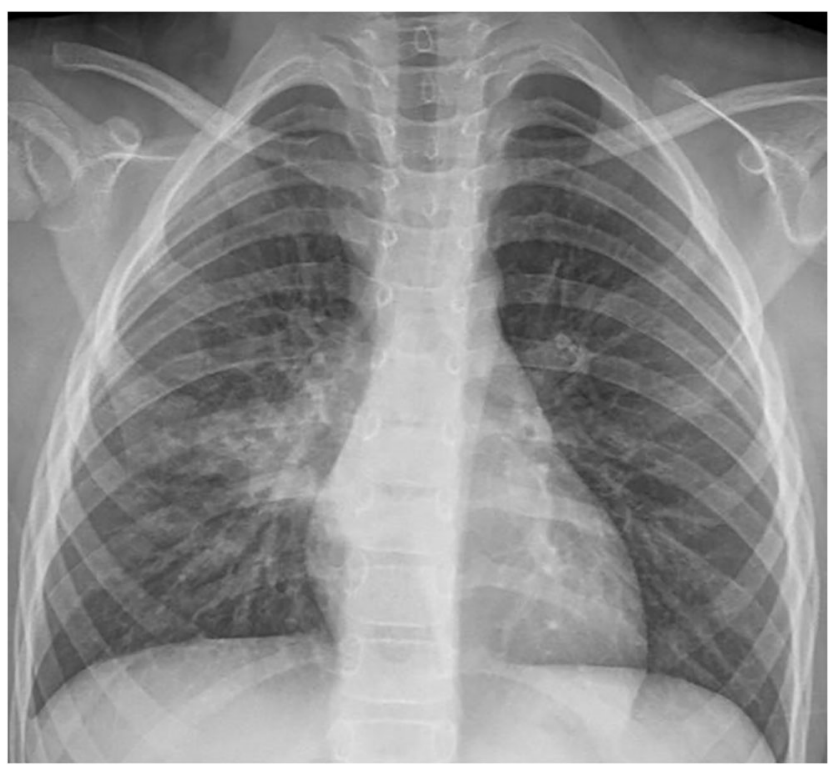

Fig. 6 Vascular engorgement in a 5-year-old girl, previously healthy, with fever for 3 days and clinical suspicion of viral infection of the lower respiratory tract. Posteroanterior chest radiograph shows vascular engorgement, more evident on the right side, with right perihilar consolidation and ground-glass opacities

on the chest radiograph in $63.7 \%$ of cases. For four children with vascular engorgement on initial radiograph, the progression to ground-glass opacity or consolidation was confirmed in follow-up radiograph or CT (Figs. 8 and 9).

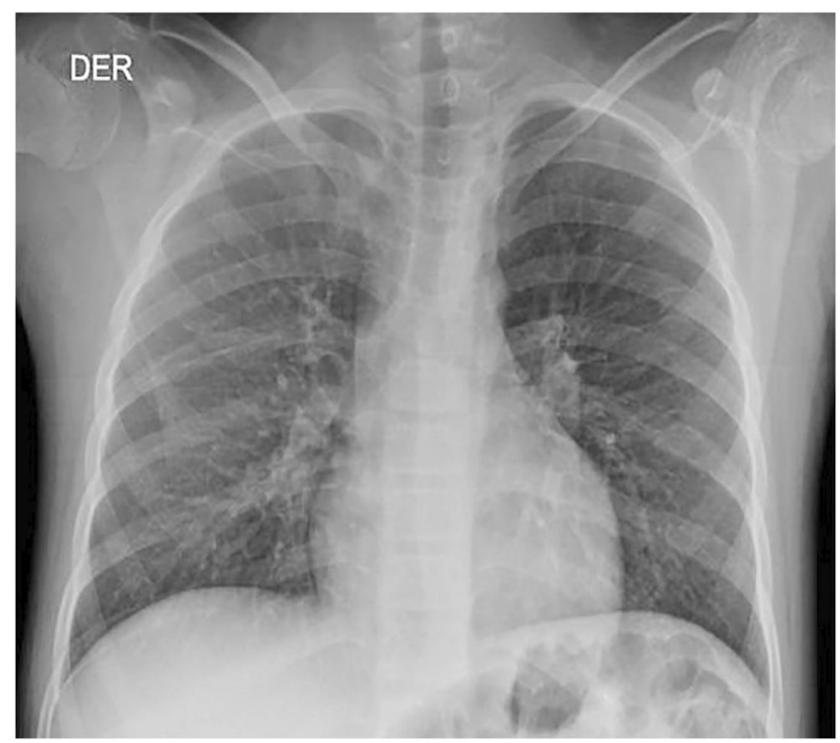

Fig. 7 Vascular engorgement in a 12-year-old asymptomatic boy who had a positive reverse-transcriptase polymerase chain reaction (RT-PCR) test for SARS-CoV-2. This image was acquired within the first $72 \mathrm{~h}$ of clinical suspicion. Anteroposterior chest radiograph shows bilateral vascular engorgement

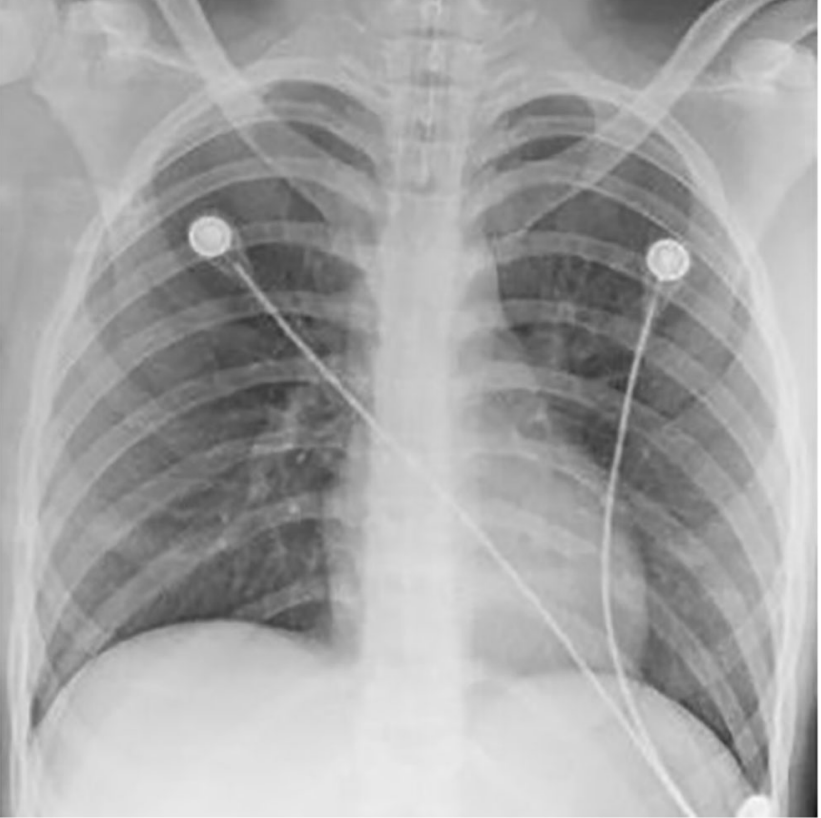

Fig. 8 Vascular engorgement in a 13-year-old girl, previously healthy, 1 day after fever onset. She had $\mathrm{O}_{2}$ saturation of $89 \%$ and required intensive care unit (ICU) admission. At ICU admission, an anteroposterior chest radiograph shows subtle right lower lobe vascular engorgement

\section{Chest computed tomography}

Chest CT was performed in 32 children in our cohort, on average $8.8 \pm 10.1$ days after the onset of symptoms. The most frequent CT findings were ground-glass opacity $(n=29$;

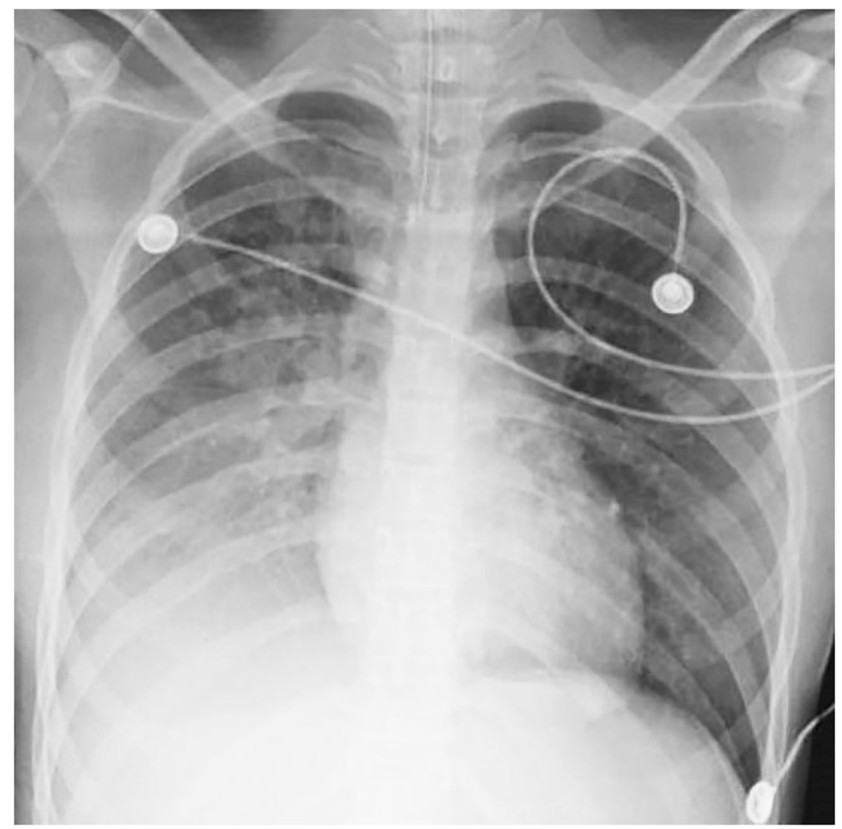

Fig. 9 Consolidation in a 13-year-old girl (same girl as in Fig. 8), $24 \mathrm{~h}$ post-admission to the intensive care unit. Anteroposterior chest radiograph shows diffuse consolidation in the right lower lobe and right central ground-glass opacity. Her clinical status worsened and chest CT was performed (not shown) 
Table 3 Chest radiographic and CT findings frequencies (\%) by clinical characteristics

\begin{tabular}{|c|c|c|c|c|c|c|c|c|c|}
\hline & \multicolumn{3}{|c|}{ Age group (years) } & \multicolumn{2}{|c|}{ Symptomatic } & \multicolumn{2}{|c|}{ ICU admission or death } & \multicolumn{2}{|c|}{ Days from symptom onset } \\
\hline & 0 to $<6$ & 6 to $<13$ & $\geq 13$ & Yes & No & Yes & No & $<7$ days & $\geq 7$ days \\
\hline Radiographic features $n(\%)$ & $n=63$ & $n=38$ & $n=30$ & $n=107$ & $n=24$ & $n=48$ & $n=83$ & $n=113$ & $n=18$ \\
\hline Vascular engorgement ${ }^{\mathrm{a}}$ & $34(54.0)$ & $30(79.0)$ & $18(60.0)$ & $44(58.9)$ & $19(79.2)$ & $24(50.0)$ & $58(69.9)$ & $72(63.7)$ & $10(55.6)$ \\
\hline Peribronchial thickening $^{\mathrm{a}}$ & $58(92.1)$ & $37(97.4)$ & $27(90.0)$ & $98(91.6)$ & $24(100)$ & $41(85.4)$ & $81(97.6)$ & $105(92.9)$ & 17 (94.4) \\
\hline Ground-glass opacity $^{\mathrm{a}}$ & $56(88.9)$ & $27(71.1)$ & $20(66.7)$ & $83(77.6)$ & $20(83.3)$ & 44 (91.7) & $59(71.1)$ & $89(78.8)$ & $14(77.8)$ \\
\hline Consolidation $^{\mathrm{a}}$ & $15(23.8)$ & $12(31.6)$ & $16(53.3)$ & $40(37.4)$ & $3(12.5)$ & 23 (47.9) & $20(24.1)$ & $32(28.3)$ & $11(61.1)$ \\
\hline CT features $n(\%)$ & $n=9$ & $n=11$ & $n=12$ & $n=30$ & $n=2$ & $n=17$ & $n=15$ & $n=16$ & $n=16$ \\
\hline Vascular engorgement & $7(77.8)$ & $11(100)$ & $9(75.0)$ & $25(83.3)$ & $2(100)$ & $14(82.4)$ & $13(86.7)$ & $15(93.8)$ & $12(75.0)$ \\
\hline Peribronchial thickening & $6(66.7)$ & $8(72.7)$ & $9(75.0)$ & $21(70)$ & $2(100)$ & $11(73.3)$ & $12(70.6)$ & $12(75.0 \%)$ & $11(68.8)$ \\
\hline Ground-glass opacity & $9(100)$ & $10(90.9)$ & $10(83.3)$ & $27(90.0)$ & $2(100)$ & $16(94.1)$ & $13(86.7)$ & $15(93.8)$ & $14(87.5)$ \\
\hline Crazy paving & $2(22.2)$ & $3(27.3)$ & $5(41.7)$ & $10(33.3)$ & $0(0.0)$ & $6(35.3)$ & $4(26.7)$ & $4(25.0)$ & $6(37.5)$ \\
\hline Consolidation & $6(66.7)$ & $7(63.6)$ & $9(75.0)$ & $21(70.0)$ & $1(50.0)$ & $12(70.6)$ & $10(66.7)$ & $11(68.8)$ & $11(68.8)$ \\
\hline Halo & $1(11.1)$ & $5(45.5)$ & $6(50.0)$ & $12(40.0)$ & $0(0.0)$ & $6(35.3)$ & $6(40.0)$ & $7(43.8)$ & $5(31.3)$ \\
\hline Reverse halo & $3(33.3)$ & $2(18.2)$ & $6(50.0)$ & $11(36.7)$ & $0(0.0)$ & $8(47.1)$ & $3(20.0)$ & $4(25.0)$ & $7(43.7)$ \\
\hline
\end{tabular}

$I C U$ intensive care unit, $n$ number of patients

${ }^{a}$ Bold font indicates $P<0.05$, significant with the Fisher exact or chi-square test

90.6\%) and vascular engorgement $(n=27 ; 84.4 \%)$ (Table 1$)$. Ground-glass opacity was characteristically bilateral (79\%), diffuse (76\%) and with segmental (48\%) and subpleural distributions (45\%) (Fig. 10). Ill-defined ground-glass nodules were observed infrequently (Fig. 11). Regarding CT findings by age group, ground-glass opacity was present in $100 \%$ of preschoolers, $90.9 \%$ of the elementary school age group and in $83.3 \%$ of the teenagers (Table 3). Vascular engorgement was found characteristically in the lower lobes (100\%) and bilaterally $(84 \%)$.

Central peribronchial thickening was found in $71.9 \%$ $(n=23)$ of the CTs. Consolidation was identified in $68.8 \%$ $(n=22)$ of CT images, affecting two pulmonary lobes on average. Halo signs, reverse halo signs (Fig. 12) and crazypaving pattern were present in $37.5 \%, 34.4 \%$ and $31.3 \%$ of CTs, respectively (Table 1). Consolidation, halo signs, reverse halo signs and crazy-paving signs were more frequent in teenagers, representing $75.0 \%, 50.0 \%, 50.0 \%$ and $41.7 \%$, respectively (Table 3 ). In our case series, $28 / 32$ (87.5\%) children had an unenhanced $\mathrm{CT}$, and in the 4 cases with intravenous contrast-enhanced CT, no pulmonary embolism or thrombosis was observed.

\section{Interobserver agreement}

All agreement measures (kappa statistic) for the four radiologic signs and eight $\mathrm{CT}$ signs ranged between 0.13 and 0.58 $(P<0.05)$ indicating slight to moderate agreement [11] among all reviewers. The sign with the poorest observed agreement was radiographic vascular engorgement (58.9\%), whereas radiographic peribronchial thickening showed the greatest agreement (88.8\%) (Table 4).

\section{Discussion}

Chest radiography is the recommended initial modality to evaluate the pulmonary effects of COVID-19 infection in children, and our study revealed that all radiographs of children with proven COVID-19 infection were abnormal. The first published studies of children $[13,14]$ reported few findings in radiographs; however, subsequent reports [9] highlighted findings that had probably not been considered, such as peribronchial thickening. It is known that intra- and interobserver variability account for differences in varying observer expertise in the imaging interpretation of pediatric pneumonia [15]. In our cohort, peribronchial thickening was the most frequent finding in radiographs, consistent with the study conducted by Caro-Dominguez et al. [9]. This finding reflects airway involvement secondary to infection with the SARSCoV-2 virus and airway inflammation. The anatomical characteristics of the tracheobronchial tree in children, with shorter and narrower airway branches, promote greater small particle and microorganism deposition in the respiratory tract as compared to adults [16]. Many other viruses and inflammatory diseases besides COVID-19 lead to central peribronchial thickening; therefore, this is a nonspecific radiologic sign of limited diagnostic value. The feature was present in all age groups and was also identified by CT (72\%). Chen et al. [6] reported that $\mathrm{CT}$ bronchial wall thickening was more commonly seen in children than in adults. 
The SARS-CoV-2 virus infects the host by binding its surface protein $\mathrm{S}$ to angiotensin converting enzyme 2 (ACE-2) receptors in alveolar cells, and also in cells of the intestine, kidney, heart and vascular endothelium [17]. In the parenchymal lung, COVID-19 causes alveolar epithelium damage, and variable degrees of exudation into the air space, which is manifested as ground-glass opacity, consolidation or crazy paving. In our case series, ground-glass opacity was the second most frequent finding in radiographs and the most frequent feature on CT $(91 \%)$. The most common ground-glass opacity distribution was bilateral and peripheral, with lower lobe predominance, which agrees with previous studies [18-20]. Ground-glass opacity was most common in children younger than 6 years $(88.9 \%, P<0.05)$. This pattern was also most frequent during the first week of SARS-CoV-2 infection and was associated with an unfavorable disease course.

Consolidation was most frequently seen in teenagers (53\%, $P<0.05)$, with unilateral and peripheral distribution. However, it was also identified in asymptomatic children (10\%) and increased in frequency and extension in children with more than 7 days of known COVID-19 infection and in children with unfavorable course.

Our study has demonstrated that imaging of asymptomatic children can show findings of active infectious disease. In our cohort, $17.9 \%$ of children who were asymptomatic but produced positive RT-PCR tests had peribronchial thickening, and many had ground-glass opacity and pulmonary vascular engorgement. Consolidation was found significantly less frequently in this group than in symptomatic children.

In our study, 32 children were evaluated with chest CT, most at 7 days after clinical onset. The main finding was ground-glass opacity, in agreement with previously published data [21-27]. In contrast with our findings, some authors, also based on pediatric CT findings, reported more localized

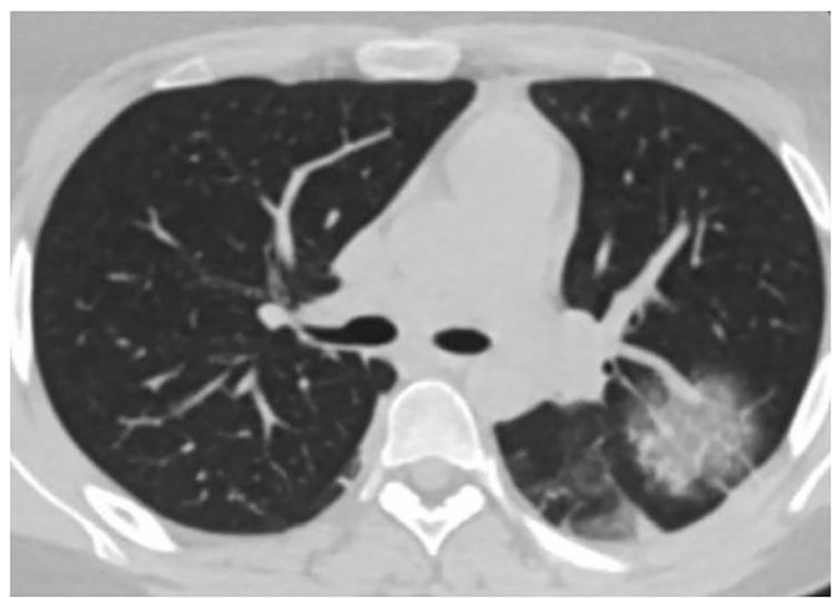

Fig. 10 Ground-glass opacities on CT in a 15-year-old boy with leukemia. The boy presented with diarrhea (suspicion of neutropenic colitis) and clinical deterioration. Axial lung window CT image shows rounded area of ground glass in the posterior segment of the left upper lobe, with prominent vessels

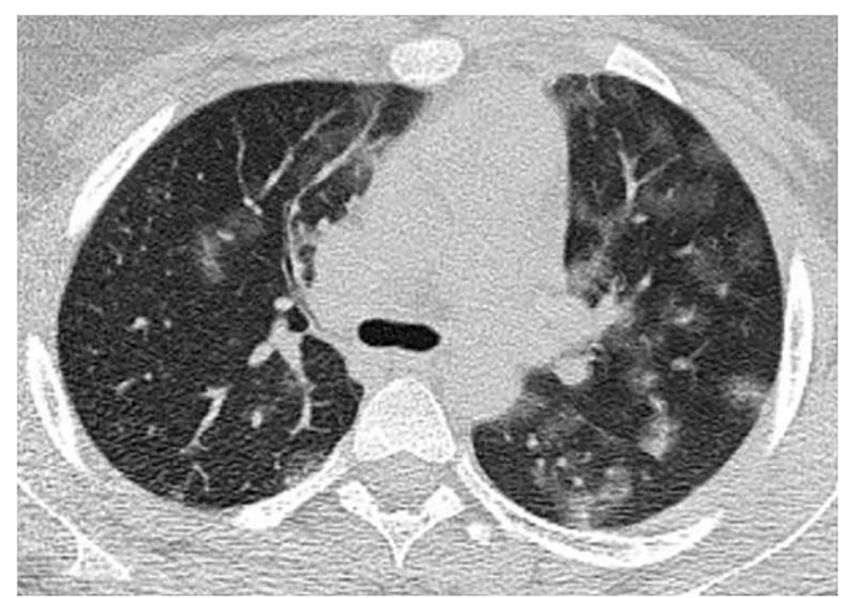

Fig. 11 Ground-glass nodules on CT in a 10-year-old boy, previously healthy, who presented with fever, dry cough, tachypnea and dyspnea that lasted for 3 days. Axial lung window CT image shows multifocal pneumonia with multiple ground-glass nodules

ground-glass opacity, lower ground-glass attenuation and lower lobar involvement in pediatric studies [24]. Some of our cases were associated with uncharacteristic imaging patterns, such as pseudo-nodular ground-glass opacity or consolidation, opacities with the peribronchovascular bundles (bronchopneumonic pattern) or manifested as ill-defined ground-glass nodules. These imaging features might be less common findings or represent early stages of pulmonary compromise in SARS-CoV-2 infection, previous to consolidation. Nevertheless, we could not rule out concurrent bacterial or viral infections.

In many studies $[8,9,19]$, ground-glass opacity has been associated with consolidation areas, halo signs or reverse halo signs. These findings were also present in our participants, mainly in teenagers. In contrast with Xia et al. [8], who suggested that the halo sign might be considered a typical sign in

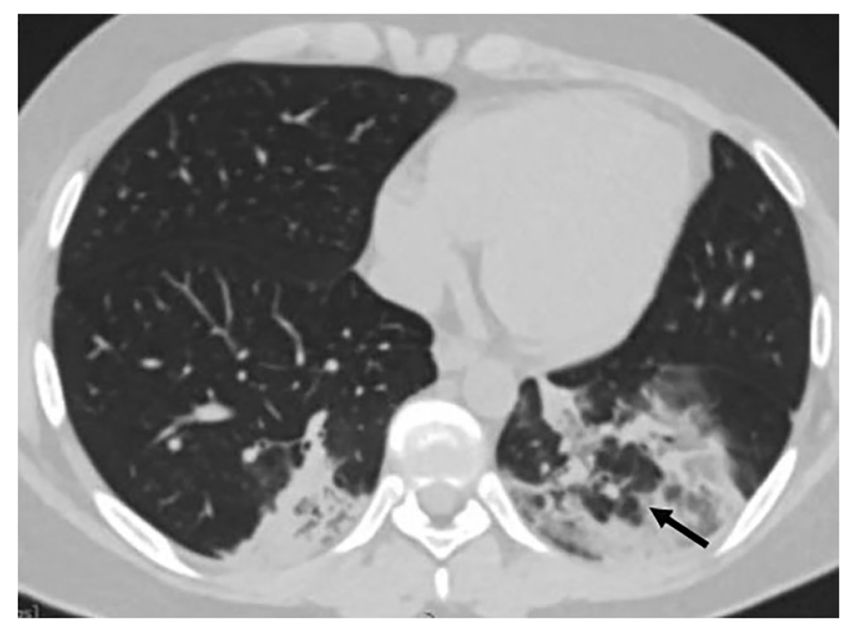

Fig. 12 Reverse halo sign in a 15-year-old boy (same boy as in Fig. 10). Axial lung window CT shows focal peripheral consolidations in the lower lobes, with a reverse halo sign (arrow) containing a dilated vessel 
Table 4 Imaging finding frequencies among raters and inter-rater agreement

Frequency, \%

Overall frequency $(95 \% \mathrm{CI}) \geq 2$ raters
Inter-rater agreement

Agreement $\% \quad$ Fleiss kappa $(95 \% \mathrm{CI}) \quad P$-value

\begin{tabular}{|c|c|c|c|c|c|c|c|}
\hline \multicolumn{8}{|l|}{ Radiographic features } \\
\hline Ground-glass opacity & $78.6(70.7-84.9)$ & 80.9 & 64.9 & 67.2 & 67.9 & $0.22(0.09-0.36)$ & 0.001 \\
\hline Vascular engorgement & $62.6(53.9-70.5)$ & 29.0 & 84.7 & 62.6 & 58.9 & $0.13(0.04-0.26)$ & 0.01 \\
\hline Peribronchial thickening & $93.1(87.2-96.4)$ & 95.4 & 87.8 & 90.1 & 88.8 & $0.31(0.12-0.49)$ & 0.001 \\
\hline Consolidation & $32.8(25.2-41.4)$ & 30.0 & 32.8 & 38.2 & 80.2 & $0.56(0.44-0.67)$ & $<0.001$ \\
\hline \multicolumn{8}{|l|}{ CT features } \\
\hline Ground-glass opacity & $90.6(73.3-97.1)$ & 68.8 & 90.6 & 93.8 & 79.2 & $0.58(0.36-0.80)$ & $<0.001^{\mathrm{a}}$ \\
\hline Crazy paving & $31.2(18.0-48.6)$ & 29.1 & 18.8 & 59.4 & 66.7 & $0.27(0.01-0.53)$ & 0.039 \\
\hline Vascular engorgement & $84.4(66.3-93.7)$ & 68.8 & 78.1 & 93.8 & 64.6 & $0.29(0.05-0.53)$ & $0.021^{\mathrm{a}}$ \\
\hline Peribronchial thickening & $71.9(53.1-85.2)$ & 65.6 & 90.6 & 56.3 & 68.9 & $0.38(0.13-0.62)$ & $0.004^{\mathrm{a}}$ \\
\hline Halo & $37.5(22.0-56.1)$ & 18.8 & 56.3 & 46.9 & 68.9 & $0.35(0.10-0.60)$ & 0.008 \\
\hline Reverse halo & $34.4(19.5-53.1)$ & 31.3 & 18.8 & 59.4 & 70.1 & $0.37(0.12-0.62)$ & 0.006 \\
\hline Consolidation & $68.8(50.0-82.9)$ & 46.9 & 68.8 & 78.1 & 75.0 & $0.45(0.19-0.71)$ & 0.001 \\
\hline Linear opacities & $15.6(6.3-33.7)$ & 25.0 & 18.8 & 18.8 & 85.4 & $0.52(0.20-0.84)$ & 0.002 \\
\hline
\end{tabular}

CI confidence interval

${ }^{a}$ Brennan-Prediger kappa coefficient. Otherwise all are Fleiss kappa coefficient

pediatric patients with COVID-19, we did not identify significant statistical data to support this hypothesis.

During radiographic evaluation, we found dilatation of the peripheral pulmonary vessels in nearly $63 \%$ of our cases. Some recently published studies have reported vascular engorgement in COVID-19; almost all were focused on adult chest CT findings [28-30]. In agreement with these reports, in our CT series we found this feature in $84 \%$ of cases. To the best of our knowledge, this finding has not been reported in radiography of pediatric patients. To further study the features of this finding, the evaluators excluded all participants with congenital heart disease or other comorbid conditions that might cause pulmonary overflow and mild vascular dilatation. Also, it should be mentioned that none of the submitted cases had myocarditis or MIS-C. The evaluator consensus resulted in 25 children with vascular engorgement as the predominant radiographic pattern. In this group of children, the feature was seen predominantly in the lower lobes, had a bilateral distribution, and was identified as isolated or in association with perihilar peribronchial wall thickening. Nevertheless, this finding had the poorest agreement among our evaluators. In addition, identifying this finding might be a challenge for radiologists with little experience in evaluating pediatric chest imaging studies.

Despite widespread interest in the pathophysiology of SARS-CoV-2 infection, there is little published evidence regarding changes in peripheral lung vessels in pediatric patients. Recently, Lang et al. [29] suggested that abnormal pulmonary vasoregulation could play an important role in COVID-19 and concluded that the dilatation of medium to small vessels, including subpleural vessels, might occur throughout the lung (not only in compromised areas), which could indicate a diffuse vascular process. Using a parallel rationale, we suggest that the pulmonary vascular engorgement found in initial radiographs reflects changes in lung parenchymal vasculature at an early stage of COVID-19 in children (Figs.1, 6 and 8).

Some postmortem studies have highlighted the diseaserelated vascular changes as a distinctive feature of SARSCoV-2 infection [31]. Damage to the vascular endothelium has been described and has been associated with the intracellular presence of the virus, distal thrombi in small pulmonary vessels, and angiogenesis [17, 31]. Cavagna et al. [32] argued that hypercoagulability associated with SARS-CoV-2 infection and concomitant endothelial damage might have an important role in pulmonary vessel damage and thrombosis. Nevertheless, validation studies are needed to thoroughly corroborate the pulmonary vascular changes and damage in children with COVID-19.

Similar to previous reports, some less common findings in our case series were pleural effusion, atelectasis, nodules and linear opacities. Lymphadenopathies were not observed.

Thoracic imaging findings certainly depend on the progress of COVID-19. It is well known that initial chest CT is normal in approximately $40 \%$ of mild or asymptomatic COVID-19 cases [33, 34]. In adult CTs, the most frequent finding is the ground-glass opacity, mainly in the second week of the disease $[25,26]$. In our cohort, a high number of cases showed a mixed pattern of ground-glass opacity, consolidation, pulmonary vascular dilatation and peribronchial thickening, probably because $\mathrm{CT}$ was performed only in very severe and 
advanced cases (more than 7 days from symptom onset) and a high percentage of children had an underlying comorbidity $(65.7 \%)$. Socioeconomic factors should also be considered in Latin America, where difficulty in accessing the health system and inequity are unfortunately common [35].

Our study has several limitations. First, it is a multicenter study with heterogeneous data sources and some incomplete clinical data. Second, different imaging acquisition protocols would impact the heterogeneous quality of the exams. Third, concurrent viral or bacterial infections could not be entirely excluded in our case series. Fourth, pediatric radiologists were not blinded to the COVID-19 diagnosis, so observer bias might be present. Fifth, in the clinical data survey, information on intravenous fluid therapy was not requested, potentially conditioning bias in the vascular engorgement finding in some children. Finally, the variable timing of imaging in relation to the presentation or course of the disease is an additional limitation of our study. Despite these limitations, this study helps establish the radiologic profile and evolution of COVID-19 in children.

\section{Conclusion}

As previously reported, COVID-19 radiographic findings in children cannot be distinguished from other airway infections. In our series, none of the studies was normal and imaging of asymptomatic children showed findings of active infectious disease. Asymptomatic children and those with mild symptoms showed mainly peribronchial thickening, vascular engorgement and ground-glass opacities. Ground-glass opacities were most frequently seen in younger children, while consolidation was most frequently observed in teenagers, and both findings were associated with unfavorable outcomes.

Supplementary Information The online version contains supplementary material available at https://doi.org/10.1007/s00247-021-05055-2.

Acknowledgments Collaborators of SLARP: Nataly Angulo, Ricardo Bado, Miriam Capetti Porto, Maria Citlaly Vasquez, Sandra Ferrón, Andrés Frosch, Mónica Galeano, Laura Gonzales, Lidia Landa, María Antonieta Londoño, Rodrigo Regacini, Samuel Sanchez, Marcelo Strauss Takahashi, Luis Torres.

\section{Declarations}

Conflicts of interest None

\section{References}

1. Palacios Cruz M, Santos E, Velázquez Cervantes MA, León Juárez M (2020) COVID-19, una emergencia de salud pública mundial [COVID-19, a worldwide public health emergency]. Rev Clin Esp 221:55-61
2. World Health Organization (2021) WHO coronavirus disease (COVID-19) dashboard. Data last updated: 2021/1/13, 9:47am CET. https://covid19.who.int/. Accessed 13 Jan 2021

3. Dong Y, Mo X, Hu Y, Qi X (2020) Epidemiological characteristics of 2,143 pediatric patients with 2019 coronavirus disease in China. Pediatrics 145:e20200702

4. Blumfield E, Levin TL (2020) COVID-19 in pediatric patients: a case series from the Bronx, NY. Pediatr Radiol 50:1369-1374

5. Levin M (2020) Childhood multisystem inflammatory syndrome — a new challenge in the pandemic. N Engl J Med 383:393-395

6. Chen A, Huang J, Liao Y et al (2020) Differences in clinical and imaging presentation of pediatric patients with COVID-19 in comparison with adults. Radiol Cardiothorac Imaging 2:e200117

7. Liu H, Liu F, Li J et al (2020) Clinical and CT imaging features of the COVID-19 pneumonia: focus on pregnant women and children. J Inf Secur 80:e7-e13

8. Xia W, Shao J, Guo Y et al (2020) Clinical and CT features in pediatric patients with COVID-19 infection: different points from adults. Pediatr Pulmonol 55:1169-1174

9. Caro-Dominguez P, Shelmerdine SC, Toso S et al (2020) Thoracic imaging of coronavirus disease 2019 (COVID-19) in children: a series of 91 cases. Pediatr Radiol 50:1354-1368

10. Hansell DM, Bankier AA, MacMahon H et al (2008) Fleischner Society: glossary of terms for thoracic imaging. Radiology 246 : 697-722

11. Feinstein AR, Cicchetti DV (1990) High agreement but low kappa: I. The problems of two paradoxes. J Clin Epidemiol 43:543-549

12. Brennan RL, Prediger DJ (1981) Coefficient kappa: some uses, misuses, and alternatives. Educ Psychol Meas 41:687-699

13. Qiu H, Wu J, Hong L et al (2020) Clinical and epidemiological features of 36 children with coronavirus disease 2019 (COVID19) in Zhejiang, China: an observational cohort study. Lancet Infect Dis 20:689-696

14. Biko DM, Ramirez-Suarez KI, Barrera CA et al (2020) Imaging of children with COVID-19: experience from a tertiary children's hospital in the United States. Pediatr Radiol 51:239-247

15. Neuman MI, Lee EY, Bixby S et al (2012) Variability in the interpretation of chest radiographs for the diagnosis of pneumonia in children. J Hosp Med 7:294-298

16. Deng Q, Ou C, Chen J, Xiang Y (2018) Particle deposition in tracheobronchial airways of an infant, child and adult. Sci Total Environ 612:339-346

17. Varga Z, Flammer AJ, Steiger P et al (2020) Endothelial cell infection and endotheliitis in Covid-19. Lancet 395:1417-1418

18. Lai W, Xie C, Pan H et al (2020) Computed tomography of the lungs in novel coronavirus (COVID-19) infection. Pediatr Radiol 50:1016-1017

19. Steinberger S, Lin B, Bernheim A et al (2020) CT features of coronavirus disease (COVID-19) in 30 pediatric patients. AJR Am J Roentgenol 215:1-9

20. Wang Y, Dong C, Hu Y et al (2020) Temporal changes of CT findings in 90 patients with COVID-19 pneumonia: a longitudinal study. Radiology 296:E55-E64

21. Berheim A, Mei X, Huang $M$ et al (2020) Chest CT findings in coronavirus disease-19 (Covid-19): relationship to duration of infection. Radiology 295:685-691

22. Kanne JP (2020) Chest CT findings in 2019 novel coronavirus (2019 n-CoV) infection from Wuhan, China: key points for the radiologist. Radiology 295:16-17

23. Kim H (2020) Outbreak of novel coronavirus (Covid-19): what is the role of radiologist? Eur Radiol 30:3266-3267

24. Duan Y, Zhu Y, Tang L, Qin J (2020) CT features of novel coronavirus pneumonia (COVID-19) in children. Eur Radiol 30: $4427-4433$ 
25. Salehi S, Abedi A, Balakrishnan S, Gholamrezanezhad A (2020) Coronavirus disease 2019 (COVID-19): a systematic review of imaging findings in 919 patients. AJR Am J Roentgenol 215:87-93

26. Pan F, Ye T, Sun P et al (2020) Time course of lung changes at chest CT during recovery from coronavirus disease 2019 (COVID19). Radiology 295:715-721

27. Kong W, Agarwal PP (2020) Chest imaging appearance of COVID-19 infection. Radiol Cardiothorac Imaging 2:e200028

28. Yin Z, Kang Z, Yang D et al (2020) A comparison of clinical and chest $\mathrm{CT}$ findings in patients with influenza a $(\mathrm{H} 1 \mathrm{N1})$ virus infection and coronavirus disease (COVID-19). AJR Am J Roentgenol 215:1-7

29. Lang M, Som A, Carey D et al (2020) Pulmonary vascular manifestation of Covid-19 pneumonia. Radiol Cardiothorac Imaging 2:327

30. Parry AH, Wani AH (2020) Segmental pulmonary vascular changes in COVID-19 pneumonia. AJR Am J Roentgenol 215:W33
31. Ackerman M, Verleden SE, Kuehnel M et al (2020) Pulmonary vascular endothelialitis, thrombosis and angiogenesis in Covid-19. N Engl J Med 383:120-128

32. Cavagna E, Muratore F, Ferrari F (2020) Pulmonary thromboembolism in Covid-19: venous thromboembolism or arterial thrombosis? Radiol Cardiothorac Imaging 2:4

33. Lu X, Zhang L, Du H et al (2020) SARS CoV-2 infection in children. N Engl J Med 382:1663-1665

34. Li W, Cui H, Li K et al (2020) Chest computed tomography in children with COVID-19 respiratory infection. Pediatr Radiol 50: 796-799

35. Frenk J, Gómez-Dantés O (2018) Health systems in Latin America: the search for universal health coverage. Arch Med Res 49:79-83

Publisher's note Springer Nature remains neutral with regard to jurisdictional claims in published maps and institutional affiliations. 